\title{
Nicht mehr Kind, noch nicht erwachsen: Entwicklungsbedürfnisse Jugendlicher in der Zange der Pandemie
}

Als Professor für Intensivpädagogik, Entwicklungswissenschaftler und pädagogischer Praktiker im Feld der Kinder- und Jugendhilfe hat mich die Pandemie von Beginn an vor allem bezüglich der psychosozialen und familiendynamischen Folgen beschäftigt. Zunächst im Kontext meiner eigenen Arbeit zum Schwerpunktthema (familiärer) Gewalt, später in einer interdisziplinären Arbeitsgruppe, angesiedelt am Institut für Virologie der Universitätsklinik Frankfurt und letztlich als Mitautor der „No-COVID“-Initiative in Deutschland hat mich die Frage bewegt, wie sich die Situation der Pandemie auf Familien auswirkt und welche Strategien jungen Menschen am meisten Unterstützung bieten. Dabei habe ich das Ziel verfolgt, Wege durch und aus der Pandemie heraus aufzuzeigen.

\section{Einleitung}

Die unterschiedlichen Maßnahmen, die zur Eindämmung der COVID-19 Pandemie ergriffen wurden, veränderten die Lebenssituation von Kindern und Jugendlichen grundlegend. Schulausfälle, Home-Schooling, Kontaktbeschränkungen, Social (oder besser: Physical) Distancing, Schließung von Einkaufs- und Freizeitmöglichkeiten - all dies sind effektive Maßnahmen gegen die Ausbreitung eines respiratorischen Virus wie des SARS-CoV-2 Erregers. Zugleich stellen sie einen tiefgreifenden Einschnitt für die betroffenen Menschen dar und entfalten somit neben den erwünschten auch unerwünschte Effekte. Dies ist keinesfalls eine Überraschung, sondern ein aus vergangenen Epidemien und Pandemien bekanntes psychologisches Phänomen (Taylor 2019).

Leider stellte sich in der Diskussion schnell eine Sichtweise ein, die als dualistisch bezeichnet werden muss: Unterschieden wurde auf der einen Seite zwischen den gesundheitlichen Risiken der Erkrankung COVID-19 und auf der anderen Seite den als Kollateralschäden bezeichneten psychischen Folgen, die vor allem für Kinder und Jugendliche angenommen und wesentlich den Schulschließungen zugeschrieben wurden.

Dieser Dualismus zwischen Infektionsschutz auf der einen und psychischen Problemen auf der anderen Seite greift jedoch zu kurz. In der Debatte um die psychologische Wirkung auf die junge Generation muss sowohl der Dynamik ei- 
ner Pandemie wie auch der Komplexität der menschlichen Psyche und dem System Familie Rechnung getragen werden. So können z. B. Maßnahmen wie ein so genannter Lockdown sowohl einen psychisch negativen Effekt haben (vor allem Verstärkung von Angst, Isolation und Einsamkeit) als auch Ressourcen aktivieren und von den jungen Menschen als gute Zeit erlebt werden (Gonzáles-Calvo und Arias-Carballal 2021; Baumann et al. 2021). Genauso kann eine Maßnahme, die zum Gesundheitsschutz ergriffen wird, eine schwere psychosoziale Belastung darstellen. Wenn diese Maßnahme allerdings nicht ergriffen wird, kann unter den Bedingungen einer Pandemie genauso eine schwere Schädigung eintreten. Beispielhaft kann hier der Wechsel von Präsenzunterricht in das Home-Schooling gelten. Es ist unstrittig, dass die Aussetzung von Präsenzbeschulung eine erhebliche Belastung darstellt, z. B. aufgrund fehlender Tagesstruktur, schwer vereinbarer Balance zwischen Kinderbetreuung und Erwerbstätigkeit der Eltern, mangelnder sozialer Beziehungen der Jugendlichen, Leistungsabfall aufgrund fehlender technischer Infrastruktur oder didaktisch schlecht gemachtem Distanzunterricht usw. Wenn aber trotz rasant steigender Inzidenzen die Schulen im Normalbetrieb verbleiben, löst dies ebenfalls erhebliche Probleme aus, z. B. ein erhöhtes Infektionsrisiko für Familien (inklusive erhöhter Mortalität in der Altersgruppe der Elterngeneration) sowie eine Verlängerung des Lockdowns außerhalb der Schulen, so dass schließlich das gleiche Ergebnis erreicht wird: Eine Verschärfung des Armutsrisikos für vom Lockdown betroffene Berufszweige, hohe Quarantänisierungsraten und schlussendlich auch das Gefühl der Betroffenen, nicht hinreichend vor der Erkrankung geschützt zu werden (Baumann et al. 2021; Baumann 2021).

Insofern braucht es also für die Betrachtung der psychosozialen Folgen der Pandemie eine komplexere Vorstellung.

\section{Jugend in der Pandemie - Risiken und Ressourcen}

Grundlegend kann bezüglich des Jugendalters wohl festgehalten werden: Es entspricht nicht den Bedürfnissen eines Teenagers, den ganzen Tag mit seinen Eltern (und ggf. Geschwistern) in der Wohnung der Familie zu verbringen. Der US-amerikanische Entwicklungsforscher Laurence Steinberg bemerkte sehr früh in der Pandemie, dass es das besondere Kennzeichen des Jugend- und jungen Erwachsenenalters sei, dass sich deren soziale Bedürfnisse zunehmend aus der Kernfamilie heraus verlagern und der Bezug zur Peergroup, das Experimentieren mit der eigenen Identität im Kontakt zu Gleichaltrigen und das Erleben von Gruppenzu- 
gehörigkeit immer wichtiger werden (Steinberg 2020). Insofern ist es auch wenig verwunderlich, dass vorläufige - noch unveröffentlichte - Daten der international angelegten COH-FIT Studie nach Aussagen von dem an der Studie beteiligten Kinder- und Jugendpsychiater Christoph Correll (Direktor der Klinik für Psychiatrie, Psychosomatik und Psychotherapie des Kindes- und Jugendalters der Charité Berlin) zeigen, dass gerade die im Übergang befindlichen jungen Erwachsenen am stärksten unter der Pandemie gelitten haben (Faigle et al. 2021).

Aber die Studienlage zeigt ein sehr differenziertes Bild. Eine groß angelegte internationale Review-Studie kommt zu dem Ergebnis, dass die meisten Familien mit noch minderjährigen Kindern und Jugendlichen die Phasen des Lockdowns insgesamt gut überstanden haben (Prati und Mancini 2021). Untersuchungen mit dem Fokus auf psychische Auffälligkeiten von Kindern und Jugendlichen kommen jedoch zu dem Ergebnis, dass deren Anteil gestiegen ist (Ravens-Sieberer et al. 2021). Eine Studie mit Jugendlichen in Brandenburg konnte zeigen, dass ca. $80 \%$ der teilnehmenden Jugendlichen mit ihrer Lebens-, Familien- und Freundessituation sehr zufrieden waren (Sturzbecher et al. 2021). Und auch andere Studien zeigen ein ambivalentes Bild, so dass einfache Aussagen über „die Jugend“ wohl kaum möglich erscheinen. Ich habe einmal versucht, den wissenschaftlichen Forschungsstand zu den Auswirkungen der Pandemie auf Kinder und Jugendliche in einem Modell zusammenzufassen, das versucht, die Zirkularität von Entwicklung zwischen dem Individuum, seiner (sozialen) Umwelt und der Pandemie abzubilden (vgl. Baumann 2021 - hier finden sich auch die Literaturangaben zu den Studien, die dem Modell zugrunde liegen).

\section{Ein Modell der bio-psycho-sozialen Dynamik der Pandemie}

Einerseits muss attestiert werden, dass in vielen Familien und bei vielen jungen Menschen durch die Ausnahmesituation Ressourcen freigesetzt wurden, die teilweise sogar zu einer Steigerung der Lebensqualität und des psychischen Wohlbefindens beigetragen haben. Hierzu trugen insbesondere die Veränderungen in der Akzeptanz, im Kompetenzlevel sowie in der Infrastruktur neuer Medien bei. Auch von einer veränderten Work-Life-Balance haben viele junge Menschen auch Teenager - profitiert und benennen dies als positive Veränderung. Dort, wo die Zeit, die man als Familie mehr miteinander verbracht hat als vor der Pandemie, harmonisch verlief, wurde dies von jungen Menschen auch oft als Ressource erlebt. Und letztlich ist auch in vielen Fällen die Wertschätzung und das Bewusstsein von Freundschaften und Beziehungen gestiegen, so dass der Lockdown in 
vielen Fällen im Erleben der Jugendlichen gar nicht primär zu mehr Isolation, sondern sogar zu engerem sozialen Zusammenhalt geführt hat.

Aber neben diesen Ressourcen bestehen auch erhebliche Risiken. Dabei ist es, wie bereits erwähnt, kein Widerspruch, dass dieselben jungen Menschen oder Familien die Situation einerseits als ressourcenstärkend und andererseits als erhebliche Belastung erlebt haben. Dass diese Seite der Risikofaktoren stärker differenziert wurde als die Ressourcenseite liegt nicht daran, dass sie aus Sicht des Autors wichtiger ist, sondern eher, dass Jugendliche mit erheblichen Belastungen Unterstützung brauchen, und dafür ein höheres Maß an Differenzierung notwendig erscheint.

Auf der Risikoseite sind einerseits die psychischen Schädigungen und Belastungen der Erkrankung und des Virus selbst zu nennen. Es mehren sich die Hinweise auf neurologische und psychiatrische Folgeerkrankungen nach einer COVID-19 Infektion. Auch Verluste von Angehörigen (im schlimmsten Falle einem Elternteil) oder schwere Krankheitsverläufe bei Jugendlichen selbst oder nahen Angehörigen oder Freunden können erhebliche psychische Problematiken provozieren. Und auch eine Reihe der so genannten Long COVID-Symptome wie z. B. Kopfschmerzen, Erschöpfung oder Gedächtnisprobleme (Brain Fog) haben einen hohen psychischen Impact, wenn sie den Menschen selbst oder eine wichtige Bezugsperson betreffen. Auch hat sich die Krankheit nicht gleichmäßig in der Bevölkerung ausgebreitet, sondern von Armut betroffene Bevölkerungsteile waren besonders häufig von Infektionen und vor allem schweren Krankheitsverläufen betroffen. Dies kann im Falle von Verdienstausfällen oder sogar dauerhaft eingeschränkter Arbeitsfähigkeit wiederum das Armutsrisiko einer Familie erheblich steigern.

Neben diesen Aspekten spielen aber auch Aspekte des Verhältnisses zwischen den Generationen eine erhebliche Rolle. Viele Entscheidungen, Regelungen und Aussagen im Rahmen des Pandemiemanagements waren derart widersprüchlich, dass dies bei einem Teil der Jugendlichen zu erheblicher Verunsicherung bis hin zu starker Wut geführt hat. Bei zunächst sehr hoher Akzeptanz der Pandemiemaßnahmen in der jungen Generation führte die zunehmend erlebte Ungerechtigkeit, die Reduktion auf die Rolle als Schüler:in sowie Entscheidungen, die gegen die Interessen (und z. T. auf Kosten) junger Menschen getroffen wurden, zu Unverständnis bis hin zu schweren Konflikten in Familien über Regeln und Regeleinhaltung - in alle Richtungen.

Und letztlich haben natürlich die Maßnahmen der Reduktion des sozialen Lebens selbst einen erheblichen „psychologischen Fußabdruck“ (Taylor 2019) hinterlassen. Ein wesentlicher Aspekt scheint dabei der Faktor des beruflichen bzw. wirtschaftlichen Drucks auf die Familie darzustellen. Armutsbedrohung, „sozialer Abstieg“ und Existenzängste sind bekanntermaßen ein erheblicher Risikofak- 


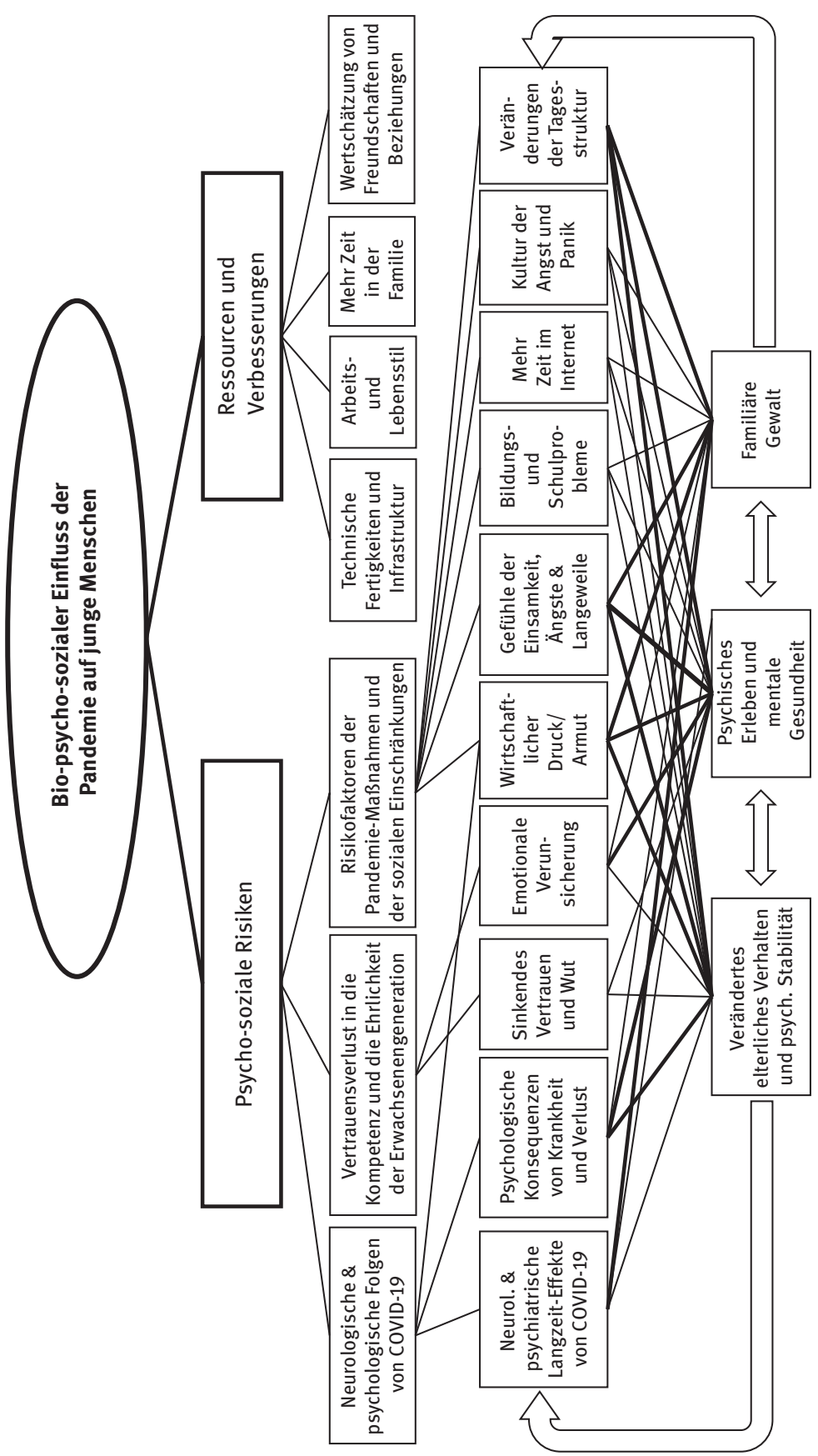

Abb. 1: Ein Modell der bio-psycho-sozialen Dynamik der Pandemie (Baumann 2021) 
tor für die Entwicklung, und dies bestätigte sich auch im Kontext der Pandemie. Auch verstärkten sich Gefühle der Einsamkeit, Ängste (bis hin zu pathologischen Ausprägungen) und der Langeweile bei einem erheblichen Teil der jungen Menschen, vor allem im Jugend- und jungen Erwachsenenalter, wo die sozialen Bezüge außerhalb der Kernfamilie elementar sind. Im schulischen Bereich konnte ein deutliches Auseinanderklaffen der Leistungsstände einzelner Jugendlicher nachgewiesen werden, was vor allem junge Menschen betraf, die schon vor der Pandemie von Bildungsbenachteiligung betroffen waren. Pädagogisch lässt sich natürlich viel darüber diskutieren, ob mehr Zeit vor Bildschirmmedien einen Risikofaktor darstellt. Im Rahmen der Pandemie kann aber festgehalten werden, dass Mobbing und sexuelle Belästigung im Internet einen starken Anstieg erlebt haben und somit ist diesbezüglich in dieser Zeit von einer Risikokonstellation auszugehen, da mehr Kinder und Jugendliche zu Opfern dieser Form der Gewalt geworden sind. Auch die Allgegenwart des Themas Corona und damit verbunden eine „Kultur der Panik“ (Fitzgerald et al. 2020) haben ihre Wirkung auf junge Menschen nicht verfehlt, vor allem wenn in sozialen Netzwerken einflussreiche Influencer ihre Krankheitsgeschichten verbreitet haben oder wenn Fake-News in die eine oder andere Richtung die Runde machten. Und schlussendlich wurde der Tagesrhythmus und die Tagesstruktur in den meisten Familien erheblich auf den Kopf gestellt. Die Auswirkungen sind zwar für jüngere Kinder gravierender, aber auch für Jugendliche sind Veränderungen täglicher Routinen sowie eine Verschiebung des Tag-Nacht-Rhythmus ein zu berücksichtigender Aspekt des psychosozialen Wohlbefindens.

Alle diese genannten Faktoren wirken letztlich wechselseitig auf drei zentrale Aspekte: Das Verhalten sowie die psychische Verarbeitung der Eltern als nach wie vor wichtige Bindungspersonen, die psychische Entwicklung und Gesundheit des jungen Menschen selbst und die Konfliktdynamik der Familie, die im ungünstigsten Fall bis zur familiären Gewalt eskalieren kann. Diese Aspekte können durch die genannten Risikofaktoren einerseits beeinflusst werden, wirken ihrerseits aber auch auf die Risikofaktoren zurück und machen einen jungen Menschen vulnerabel für die speziellen Einflüsse der Pandemie.

\section{Konsequenzen: Was brauchen Jugendliche von der Erwachsenengeneration?}

Wie in der Überschrift dieses Beitrages angedeutet, befinden sich Jugendliche in einer besonderen Situation in dieser Pandemie. Infektiologisch scheinen sie dieselbe Rolle wie Erwachsene gespielt zu haben, und so wurde von ihnen auch erwartet, erhöhte Sicherheitsmaßnahmen einhalten zu können. Dies haben sie Stu- 
dien zufolge auch größtenteils getan (Schnetzer und Hurrelmann 2020). Gleichzeitig sind Jugendliche aber gerade in der Phase, in der soziale und körperliche Distanzierung, Home-Schooling und damit einhergehende Eigenverantwortung, fehlende Möglichkeiten von Sport, Freizeit und Feiern etc. den alterstypischen Entwicklungsbedürfnissen elementar widersprechen. In der Lebensphase, in der die Abgrenzung von den Eltern, die Erfindung der eigenen sozialen Identität, erste Experimente mit Körperlichkeit, Sexualität und Intimität zentrale Entwicklungsaufgaben darstellen und auf dem Bildungsweg wichtige Weichen gestellt werden, veränderte die Pandemie die Lebenswelt radikal und engte die ausgegebene Order Stay at home die individuellen Möglichkeiten stärker ein als in jeder anderen Entwicklungsphase.

Insofern ist für jede Phase der Pandemie - zumal wenn diese so lange andauert wie die COVID-19-Pandemie in Mitteleuropa - eine Fokussierung auf die Entwicklungsbedürfnisse Jugendlicher und junger Erwachsener elementar. Dies sollte aber in dem oben skizzierten Spannungsverhältnis betrachtet werden, in dem Maßnahmen zum Infektionsschutz nicht gegen psychisches Leid aufgerechnet werden können. Dieser Dualismus führt weder weiter noch gibt er die notwendige Handlungsfähigkeit für ein Pandemiemanagement, das sich den jeweiligen dynamischen Entwicklungen anpassen kann. Ein bloßes Infragestellen der Maßnahmen, weil Menschen darunter leiden, greift dabei zu kurz (vgl. ausführlich Baumann et al. 2021).

Vier wichtige Hypothesen/Forderungen leite ich daraus zusammenfassend ab:

Die Gesellschaft sollte sich darüber im Klaren sein, dass junge Menschen legale Kontaktmöglichkeiten brauchen. Sichere Kontakte, differenzierte Regeln, wie diese gestaltet werden können und Orte, an denen sich junge Menschen unter Einhaltung von Regeln treffen dürfen, scheinen unausweichlich. Testkapazitäten, Räumlichkeiten und der aktuellen Pandemiedynamik angepasste Kontaktbudgets müssen speziell für junge Menschen freigegeben werden, um Entwicklung zu ermöglichen.

Schulen müssen den aktuellen Wissensständen gemäß zu sicheren Orten werden. Einerseits, um Präsenz auch bei dynamischer Entwicklung der Pandemie zu gewährleisten (bzw. zu verteidigen), und andererseits, um den jungen Menschen zu signalisieren, dass auch sie geschützt werden sollen. Dies betrifft neben dem Unterrichtsgeschehen gerade auch Pausenbereiche und Schulwege, z. B. den öffentlichen Personennahverkehr.

Stärken- und Ressourcenorientierung sind die Basis psychischer (Re-)Stabilisierung. Eine Konfrontation mit Defiziten (zum Beispiel Aufholen von Lernrückständen) sowie Noten- und Leistungsdruck müssen zeitlich und von der Priorisierung hinter die psychische und soziale (Re-)Integration gestellt werden. 
Ein besonderes Augenmerk muss auf vulnerable und von Benachteiligung bedrohte Gruppen gelegt werden. Hier sind besondere Maßnahmen zu entwickeln, um in jeder Pandemiephase für diese Gruppen (auch wirtschaftliche) Sicherheit, Begleitung und Förderung zu gewährleisten. Gerade auch im Nachklang der Pandemie werden diese Gruppen besondere Aufmerksamkeit und Begleitung brauchen.

\section{Literatur}

Baumann M (2021) COVID-19 and mental health in children and adolescents: a diagnostic panel to map psycho-social consequences in the pandemic context. Discover in Mental Health 1, 1. https://doi.org/10.1007/s44192-021-00002-x.

Baumann M, Berghäuser M, Bolz T, Martens T (2021) Den Fokus neu denken - Skizze eines Pandemiemanagements auf Grundlage der Bedürfnisse und Lern- und Entwicklungserfordernisse von Kindern, Jugendlichen und Familien. https://www.socialnet.de/materialien/ 29164.php. Zugriff am 1. Februar 2021.

Faigle P, Simmank J, Correll C (2021) Psyche und Corona: „Das dickere Ende kommt noch“. Zeit Online 8. Juni 2021. https://www.zeit.de/gesundheit/2021-07/psyche-corona-mentalegesundheit-pandemie-psychologie-christoph-u-correll?utm_referrer=https $\% 3 \mathrm{~A} \% 2 \mathrm{~F}$ \%2Fwww.google.com\%2F. Zugriff am 21. September 2021.

Fitzgerald DA, Nunn K, Isaacs D (2020) Consequences of physical distancing emanating from the COVID-19 pandemic. An Australian perspective. Paediatric Respiratory Reviews 35:25-30. https://doi.org/10.1016/j.prrv.2020.06.005.

Gonzáles-Calvo G, Arias-Carballal M (2021) A World Through Glass: A Narrative Around the Family Experience During the Confinement of COVID-19. Journal of Family Issues, 13. Juni 2021. https://doi.org/10.1177\%2F0192513X211024989.

Prati G, Mancini AD (2021) The psychological impact of COVID-19 pandemic lockdowns: A review and meta-analysis of longitudinal studies and natural experiments. Psychological Medicine 51(2):201-211. https://doi.org/10.1017/S0033291721000015.

Ravens-Sieberer U, Kaman A, Otto C, Erhart M, Devine J, Schlack R (2021) Impact of the COVID-19 pandemic on quality of life and mental health in children and adolescents in Germany. European Child \& Adolescent Psychiatry. https://doi.org/10.1007/s00787-02101726-5.

Schnetzer S, Hurrelmann K (2020) Jugend und Corona: Wie rücksichtsvoll verhalten sich die jungen Generationen? In: Die Studie „Junge Deutsche 2021“. DATAJOCKEY, Kempten.

Steinberg L (2020) Expecting Students to Play It Safe if Colleges Reopen Is a Fantasy. Safety plans border on delusional and could lead to outbreaks of Covid-19 among students, faculty and staff. New York Times, 15. June 2020. https://www.nytimes.com/2020/06/15/ opinion/coronavirus-college-safe.html. Zugriff am 21. September 2021.

Sturzbecher D, Dusin R, Kunze T, Bredow B, Pöge A (2020) Jugend in Brandenburg 2020. Auswirkungen der Corona-Pandemie. Institut für angewandte Familien-, Kindheits- und Jugendforschung e. V. an der Universität Potsdam (IFK). https://mbjs.brandenburg.de/ media_fast/6288/corona-jugenstudie_2020.pdf. Zugriff am 17. Februar 2021.

Taylor S (2019) The Psychology of Pandemics. Preparing for the Next Global Outbreak of Infectious Disease. Cambridge Scholars Publishing, Newcastle upon Tyne. 